\title{
Quantitative Compressor-Easing for Efficient Coherent X-ray Pulses
}

\author{
Davide Bleiner ${ }^{1 \mathrm{a}, \mathrm{b}}$ \\ ${ }^{a}$ Empa Materials \& Technology, Überlandstrasse 129, CH 8600 Dübendorf, Switzerland ; \\ bUniversity of Zurich, Dept. of Chemistry, Winterthurerstrasse 190, CH 8057, Zurich, Switzerland
}

\begin{abstract}
Tabletop plasma-driven X-ray lasers hold the promise to overcome beamtime limitation of accelerator facilities. Such unlimited access is essential for training purposes, industrial processes as well as for going beyond the "proof-of-principle" research. Tabletop X-ray lasers are demonstrated by single-pass amplified spontaneous emission (ASE) across a plasma medium by means of transient collisional excitation. The latter is either a discharge or a laser-produced plasma obtained by target material irradiation. The homogeneity of the plasma medium is crucial to minimize refraction or self-absorption.

Generation of ASE across a plasma column is dramatically improved under traveling-wave excitation (TWE). This means that a pump laser pulse irradiates a target with a certain angle of attack, instead of classical normal incidence. In the latter case hot spots and cold spots are formed. In the case of TWE a single sweep of plasma is formed in the same direction of pump pulse. The latter is optimized when the sweep propagation is close to the speed of light (i.e the ASE speed), which implies small-angle of attack. Yet, short-wavelength plasma lasing needs large-angle target irradiation, in order to increase the pump penetration into the denser plasma core. High electron density is needed for high collisional pumping rates.

The apparent tradeoff large angle (for short wavelength) vs small angle (for efficient gain) was solved by pulse shaping, i.e. pulse-front back-tilt. In fact, the TWE speed depends on the pulse-front slope (envelope of propagation front), whereas the optical penetration depth depends on the wavefront slope (envelope of phase). Pulse-front tilt was accomplished by means of pulse compressor misalignment ("easing"). The latter is the final stage of a chirped-pulse amplification (CPA) system that recompresses the stretched pulse.

In this study it was found effective to use the easing of the compressor, only if coupled with a high-magnification frontend imaging/focusing component, and that higher order terms are marginal. It is concluded that sweep speed matching should be accomplished with minimal compressor misalignment of a few degrees and maximal imaging magnification. A complete computational study by means of Fourier Optics was performed and validated experimentally.
\end{abstract}

Keywords: Fourier optics, compressor, pulse shaping, wavefront, travelling wave, X-ray laser, plasma

\section{INTRODUCTION}

Multi-Joule laser-pulses on solid targets generate compositionally-tuned hot and dense plasmas, used as gain media for short wavelength lasing, e.g. $\lambda<50 \mathrm{~nm}$, by amplified spontaneous emission (ASE) ${ }^{1}$. The ASE process is accomplished as a high-gain single-pass sweep across the plasma-column length. Such micro-plasmas are extremely attractive as stand-alone tabletop soft X-ray lasers or as amplifier modules in large-scale hard X-ray systems.

Previous studies have shown that the cutting-down of the wavelength requires large-angle of attack between the irradiated surface and the drive pulse ${ }^{2}$. The need to deposit the drive energy deep inside the expanding plasma, in regions of high electron density, i.e. for efficient collisional pumping, demands this. However a major drawback is to be mentioned in the off-normal incidence, i.e. the ASE gain-length is shortened. In fact, such drive pulse propagates mainly into rather than along the plasma medium. Further, under the normal-incidence scheme the ASE amplification front advances into expanding plasma regions that are increasingly cooler and rarified ("cold spots"), i.e. losing both population inversion as well as the gain. Ideally, a speed match between ASE propagation $\left(v_{A S E}\right)$ and the hot/dense plasma column front $\left(v_{P C F}\right)$ is

${ }^{1}$ E-mail: davide.bleiner@empa.ch phone +41 58765 4934; 
necessary. The latter is accomplished when the two are at the speed of light, i.e. $v_{A S E}=v_{P C F}=c$ (sweep-speed-matching condition). This would require the drive pulse to be delivered at small-angle of attack, i.e. grazing incidence pumping (GRIP), or even an angle of attack $\theta=0^{\circ}$ (parallel to the target surface), in order to have $v_{A S E}=v_{P C F}=c$. Obviously, a grazing-incidence pulse is highly reflected from the plasma, so the GRIP angle of attack must be increased.

Summarizing, one faces an optimization trade-off between short-wavelength generation (large-angle of attack) and highgain plasma lasing (small-angle of attack). A partial turnaround to such trade-off was introduced with transient collisional excitation $\left(\mathrm{TCE}^{3}\right)$, i.e. using two drive pulses, and carrying out traveling-wave excitation (TWE), as explained in detail previously ${ }^{4}$. TWE aims to achieve the speed matching condition. In fact, an inclined pulse-front deposition produces a sweep-delayed plasma column. The TWE speed is the apparent speed along the target length, and so for a given angle of attack $\theta$, the TWE speed is $v_{T W E}=c / \cos \theta$. Ideally, the $v_{T W E}$ must remain close to $v_{A S E}$. However, such a speed-matching condition is difficult to obtain at non-grazing angle of attack, due to plasma heterogeneity, and because the gain has an influence on the propagation speed ${ }^{5}$.

In this paper we validated a technique to overcome the discussed trade-off, by means of decoupling the TWE speed from the plasma penetration of a drive pulse. The basic idea integrates with existing chirped-pulse amplification (CPA) ${ }^{6}$ components, where a stretched-pulse is amplified and then (re-)compressed using a grating pair (compressor). Ideally, the two compressor gratings must be perfectly aligned to achieve Fourier-limited compression over the entire beam diameter, and thus pulse duration below 100 femtoseconds. If the two gratings are not perfectly aligned (here termed "compressor easing"), a residual angular dispersion "leaks-out" in the propagating "compressed" pulse. Angular dispersion causes a tilting of the pulse-front, not of the wave-front. A quantitative study of the easing process permits to determine the extent of pulse-front tilt and pulse duration broadening.

Indeed, quantitative compressor-easing helps tailoring the pulse-front tilt, which mitigates the angle of attack, and with that match TWE. The treatment is based on a linear variation (first-order), such that the electric field varies across the pulse with a relation like $E(\mathrm{x}, \mathrm{t}+\mathrm{x} \tan \theta / \mathrm{c})$. Differently from previous publications ${ }^{7}$, we speculate on higher-order effects, in order to have a more accurate speed-match, which possibly improves the plasma X-ray lasing performance.

A significant challenge, especially at higher order, is the accurate measurement of the pulse-front tilt, with simultaneous pulse duration. Spatio-temporal characterization of ultrashort pulses is significantly different whether it is carried out at low $^{8}$ or high power ${ }^{9}$. A number of workers have utilized autocorrelation, or variations of it, to obtain a metrology of the pulse tilt ${ }^{10,11}$.

Figueira et al. ${ }^{12}$ have recently discussed the limitation of the autocorrelation technique, especially when applied to singleshot high energy systems. In particular the lack of statistical robustness is highlighted. A modified methodology, i.e with delayed replica of the pulse to be measured, permits obtaining a more robust quantification of the pulse tilt. The method is highly versatile, self-calibrating, high resolution, and able to detect the presence of the pulse front tilt in unknown pulses, providing instantaneous feedback about their magnitude and signal.

Aim of this paper was to provide a quantitative assessment of compressor easing for TWE matching. We compare computational results with experimental data, which clarifies the scope of the proposed technique.

\section{METHODS}

\subsection{Computational Model}

Fourier optics addresses, within the paraxial or small aperture approximation, a wave optics calculation, as a product of transfer functions of optical elements. The wave convolution of a collection of elements, e.g. lens, grating, free-space, mirror, etc., is easily obtained as product of their Fourier transforms (FT). Therefore, calculating in the Fourier domain, simplifies the computation of the overall effects as a mere multiplication of characteristic functions. Inverting the FT back to the real space, provides the spatio-temporal characteristics of the pulse. The pulse emerging from the stretcher as input into the compressor was modelled as a chirped Gaussian beam with the following relation:

$E_{o}=\left(\frac{1}{\omega_{o}}\right) e^{\left[-\frac{\left(k_{x} \omega_{O}\right)^{2}}{4}\right]} e^{\left[\left(\omega-\omega_{O}\right)\left(\frac{1}{4 \Delta \omega}\right)^{2}\right]} e^{\left[-i \frac{\phi_{2}}{2}\left(\omega-\omega_{o}\right)^{2}\right]}$ 
The first term of eq.1 is the axially symmetric spatial profile with Gaussian distribution in the wave-vector domain. The second term is the spectrum, around the central frequency $\omega_{\mathrm{o}}$. The third term is the pulse chirp.

Fig 1 sketches-out the compressor setup: a four-grating (G1, G2, G3, G4) system combined with a folding roof-mirror (M) simplifies to three elements, since one introduces a constraint that $\mathrm{G} 1=\mathrm{G} 4$ and $\mathrm{G} 2=\mathrm{G} 3$ (pulse folding). This makes that the degrees of freedom (number of gratings to align minus 1) is one. At the front-end, the focusing element (F) is shown, which images the drive pulse to a $10-15$-mm line focus.

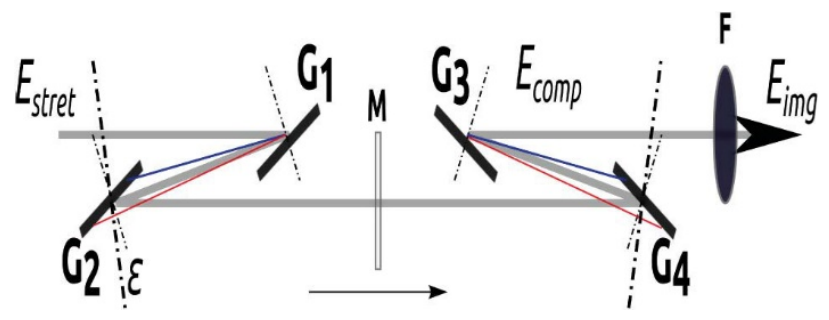

Fig.1. Experimental setup. Legend: $\mathrm{E}_{\text {stret }}$ is the chirped input pulse, G1, G2, G3, G4 are the gratings of the compressor. Due to the folding mirror M, then $\mathrm{G} 1=\mathrm{G} 3$ and G2 = G4. The tilt angle is $\varepsilon$. The focusing optics is F, and $\mathrm{E}_{\mathrm{img}}$ is the "compressed" output pulse.

The following transfer functions were adopted:

Free-space propagation (FSP): $\quad E_{F S P}=E_{\text {input }} e^{\left[-i k z-\frac{i z}{2 k} k_{x}^{2}\right]}$

with propagation in the $z$ axis where the FSP is applied on the input field $\mathrm{E}_{\text {input. }}$

Grating-dispersion (GD): $\quad E_{G D}=\frac{E_{\text {input }}}{b}$

where $b=\cos \alpha / \cos \beta_{0}$ is the beam spread, i.e. $\alpha$ is the angle of incidence on the grating, while $\beta_{o}$ is the angle of diffraction for the central frequency. Clearly the field response is a function of the spectral frequency.

The pulse focusing/imaging $(I M G): \quad \quad E_{\text {img }}=E_{\text {input }} e^{\left[\frac{i k}{2 f} r^{2}\right]}$

with the focal length $f$.

A folding roof-mirror (M), gives no effects apart from extending the free-space propagation. The convoluted system is thus the product of these Fourier terms, as shown earlier in detail ${ }^{13}$.

\subsection{Boundary Conditions and Experimental Validation}

The computations were performed for our chirped-pulse amplification (CPA) system, i.e. a Nd:glass with a wavelength of $1054 \pm 2 \mathrm{~nm}$. The Gaussian beam waist radius was $\mathrm{w}_{0}=66 \mathrm{~mm}$ and focused using a $f=609.6 \mathrm{~mm}$ spherical mirror under an angle of attack of $\theta_{\mathrm{GRIP}}=48^{\circ}$. The compressor had gratings with $1740 \mathrm{lpmm}$, and the chirped pulse stretch per bandwidth was $296 \mathrm{ps} / \mathrm{nm}$. The roof folding-mirror had a half-distance of $1360 \mathrm{~mm}$. The angle of incidence of the stretched pulse on the first grating was $60.8^{\circ}$. The imaging system had a magnification of 5:1 $(\mathrm{m}=0.2)$.Such Nd:glass CPA system was used for experimental benchmarking. The system was focused onto the target over a line-focus ${ }^{14,15}$ of $12-15 \mathrm{~mm}$ (see results for the specific values). The pulse-tilt data were obtained determining the travelling-wave speed using a streak camera connected to the leading and trailing edges of a line focus with optical fibers.

\section{RESULTS}

Fig. 2 shows calculated pulse-front delivery as a function of compressor easing. In each panel, the corresponding TWE speed is given. Firstly, an aligned compressor $\left(\zeta=0^{\circ}\right)$ is shown, which at the given angle of attack $\left(\theta_{\mathrm{GRIP}}=48^{\circ}\right)$ corresponds to a speed mismatch of $49 \%$, i.e. $\mathrm{V}_{\mathrm{Tw}} / \mathrm{c}=1.49$. Although at a first impression, the panels look very similar, a careful analysis reveals that the pulse front is back-tilted. At $\zeta=9.4^{\circ}$ one estimates a residual speed mismatch of $28 \%$, while at $\zeta=16^{\circ}$, the 
residual speed mismatch reduces to $17 \%$. The speed mismatch would be eliminated, only in the extreme case of pulse front perpendicular to the target surface, which is clearly absurd because implies no target hit. However, one can enhance the back-tilt deploying a demagnification in the focusing unit, such that length along the line-focus is shrunk. The calculated improvement from $50 \%$ to $17 \%$ (factor of 3 ) may be hindered by a concomitant broadening of the pulse length.
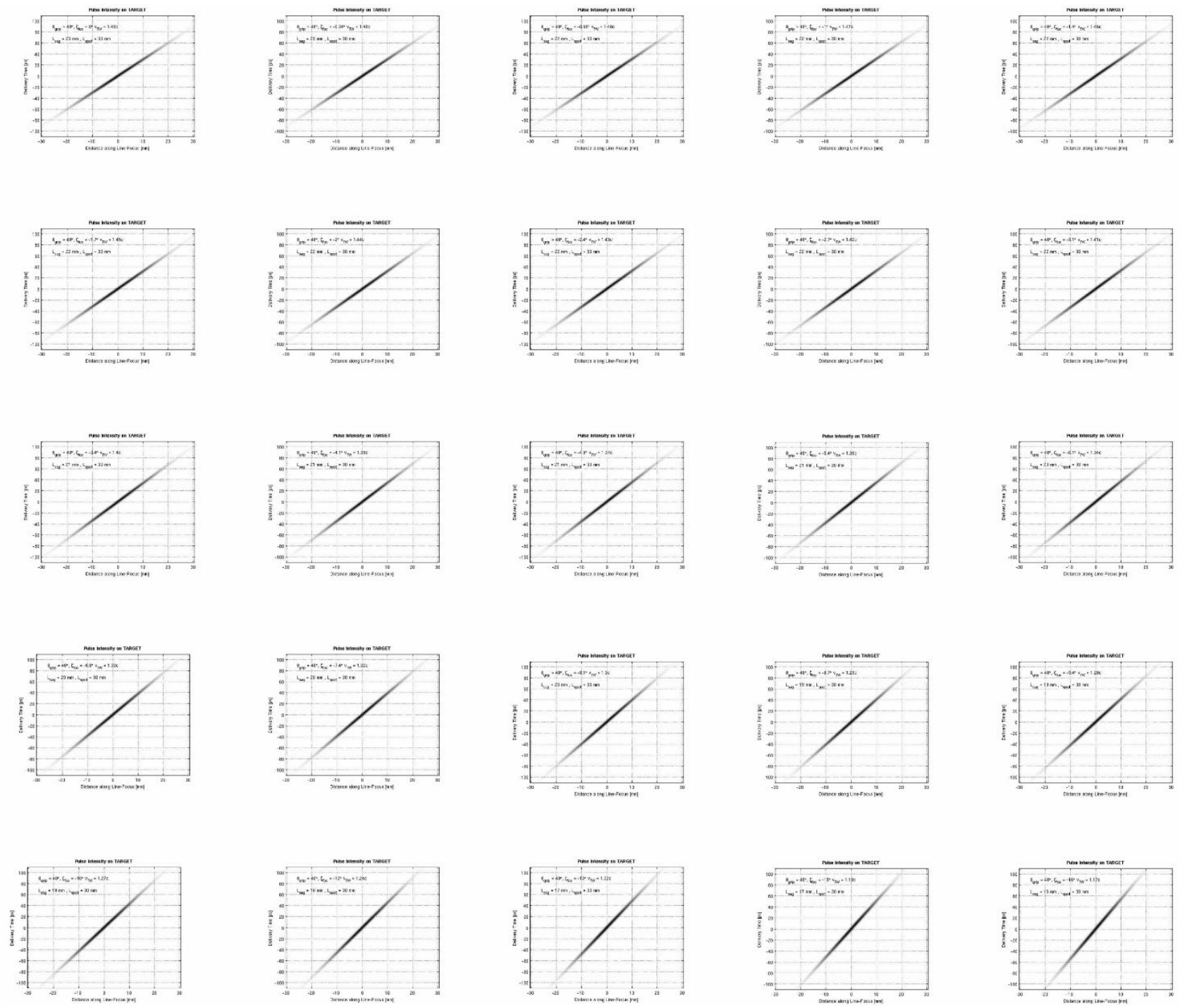

Fig. 2. Calculated pulse-front delivery as time vs. position across the line focus. The various panels show constant angle of attack $\left(\theta_{\mathrm{GRIP}}=48^{\circ}\right)$, spot length $\left(\mathrm{L}_{\mathrm{spot}}=30 \mathrm{~mm}\right)$, and variable pulse-front slope $\left(\zeta_{\mathrm{foc}}\right)$, travelling wave speed $\left(\mathrm{V}_{\mathrm{TW}} / \mathrm{c}\right)$, and sagittal focus $\left(\mathrm{L}_{\mathrm{sag}}\right)$. A careful analysis shows the small pulse-front back-tilt. Ideally $\mathrm{V}_{\mathrm{TW}}$ be as close as possible to the speed of light $\mathrm{c}$. 

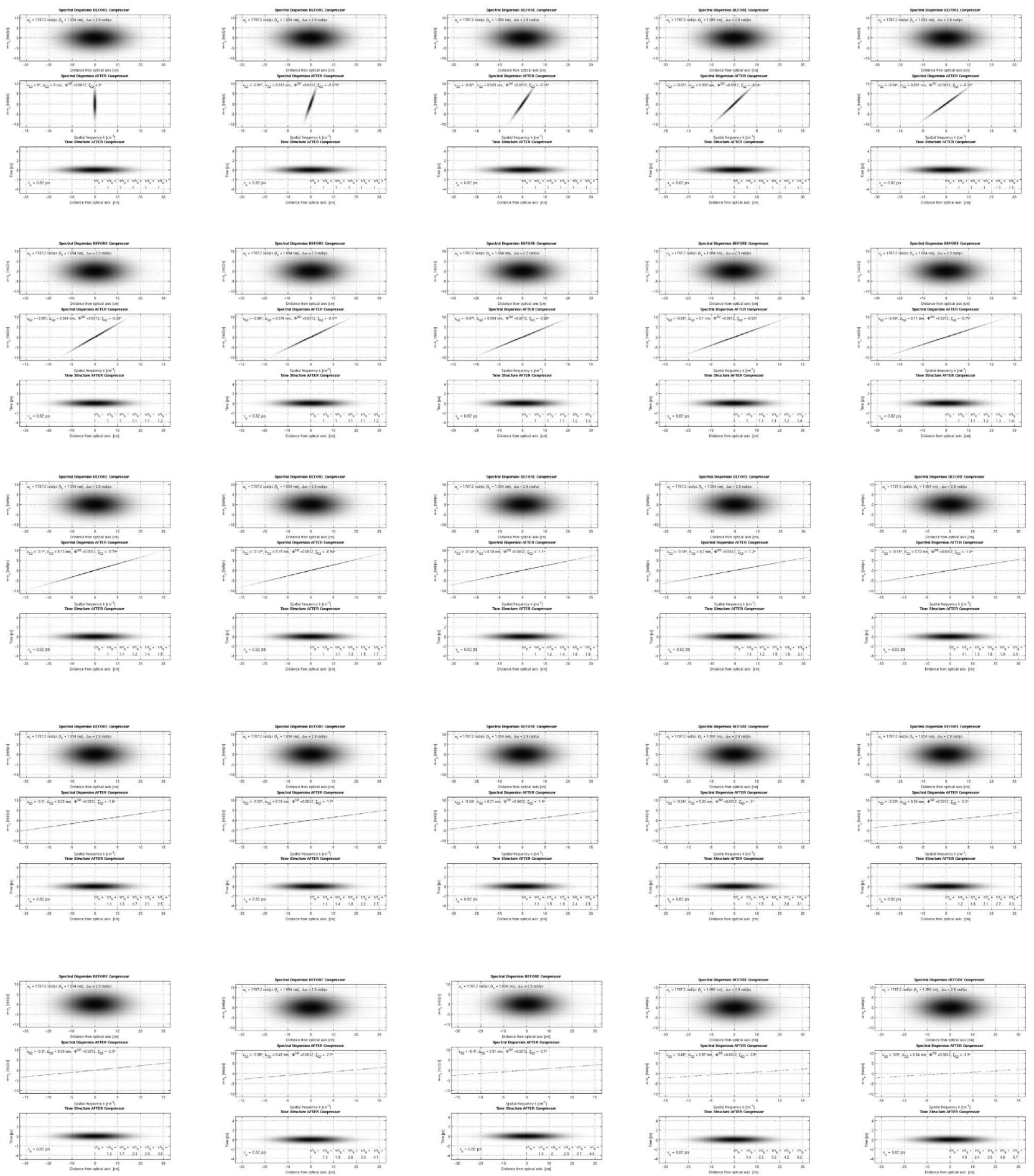

Fig. 3. Calculated dispersion during compressor easing. Each panel is composed of three subpanels, indicating (from top to bottom): (a) the spectral frequency spread across the pulse cross section. The fix parameters are the central carrier frequency $\left(\omega_{\mathrm{c}}=1787.2 \mathrm{rad} / \mathrm{ps}\right)$ corresponding to a laser wavelength of $\lambda=1^{\prime} 054 \mathrm{~nm}$, and a bandwidth of $\Delta \omega=2.9 \mathrm{rad} / \mathrm{ps}$; (b) the spectral dispersion downstream from the compressor. The changed parameters are the grating $\mathrm{G} 2$ tilt ( $\varepsilon_{\mathrm{G} 2}$ from $0^{\circ}$ to $\left.-0.50^{\circ}\right)$, the adjusted grating spacing correction $(\Delta \mathrm{G} 2$ from 0 to $0.64 \mathrm{~mm}$ ); (c) time structure after the compressor, where the pulse broadening is shown as a relative pulse increase as a factor of the fully compressed Fourier limit, i.e. $\tau_{\mathrm{o}}=0.82 \mathrm{ps}$. See text for comments. 
Fig. 3 visualizes the calculated incremental pulse broadening as a function of grating G2 easing. The uncompressed pulse is shown in the top frame of each panel (always the same). The middle frame shows the dispersion when the pulse has propagated past the compressor, as a function of compressor tilt from a fully aligned (top left) to an increasingly eased system. The spatial frequency stretch calculated in the latter cases indicated with a slanted plot, derives from a "segregation" of the frequency components across the pulse.

Each bottom frame visualizes the pulse broadening, i.e. time as function of pulse cross section. The duration is normalized to the computed Fourier-limited pulse duration at the centerline (point of best compression), i.e. $\tau_{0}=0.82 \mathrm{ps}$ (Nd:glass). One observes that the broadening, up to factor of 5.7 (bottom right panel) is concentrated at the border of the pulse. A pulse duration of $0.82 \mathrm{ps}$ is much shorter than the ASE buildup time, i.e. a few tens of ps. Therefore a G2 misalignment for pulse-front tilt has also the benefit to broaden the pumping to match the lasing dynamics. However, one has to speculate that such benefit is mainly restricted in the margin of the plasma (where the pulse broadens the most), while in the center (where hot and dense conditions are accomplished), the pulse may be still short. Ideally, one would like this dependence to be moderate, in order to prevent drastic changes in pulse peak power across the beam diameter.

\subsection{Experimental Validation}

Fig. 4.a summarizes schematically the pulse delivery concept. The determination was done at attenuated energy to prevent damage to the fiber termination. Therefore, no plasma was generated. However, the experiment served as a reference to assess the speed of apparent propagation along the target surface.

a)

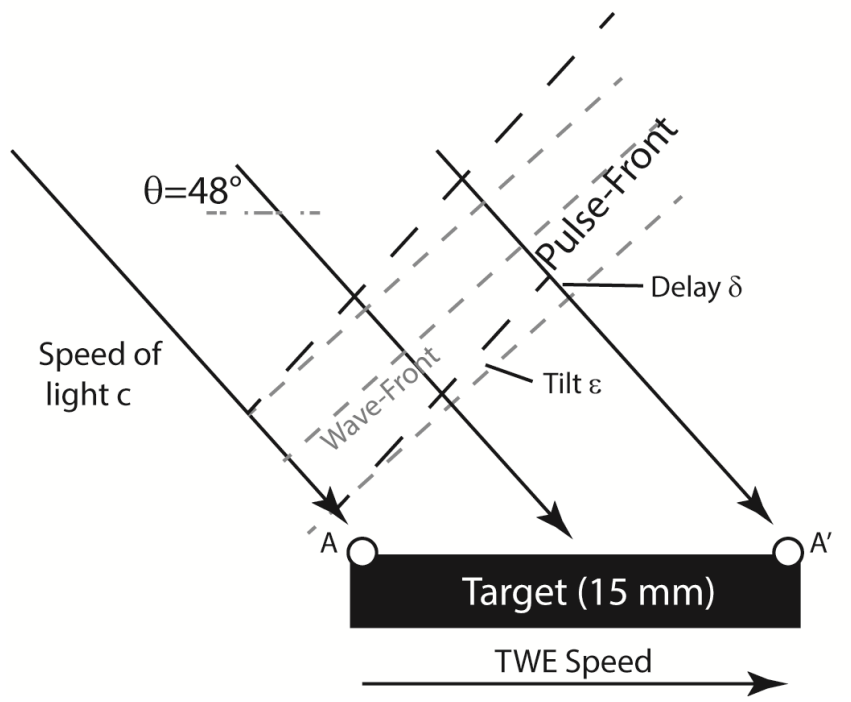

b)

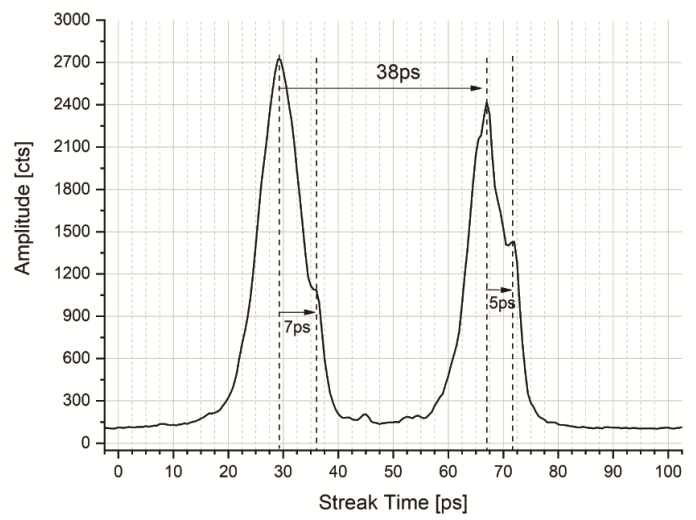

Fig. 4. (a) Schematic representation of the experimental setup, to determine the effective travelling wave speed. Two optical fibers terminations A and A', connected to a streak camera, get the start and the end signals, with $15 \mathrm{~mm}$ separation, whose delay is influenced by the pulse-front back-tilt as obliquity. (b) Experimental profiles of the streaked signals at A and A', showing an effective travel time of 38 ps.

Fig. 4.b shows, the traces of the streak camera signals. Considering an angle of attack of $\theta=48^{\circ}$ and a travel distance AA' $\Delta \mathrm{x}=15 \mathrm{~mm}$, one obtains a theoretical travel time (i.e. assuming no tilt) as follows:

$\Delta t=\frac{\Delta x}{V_{T W}}=\frac{\Delta x}{c} \cos \theta$ 
which gives $\Delta t=33.6 p s$. From Fig. 4.b one however obtains a travel time of $38 \mathrm{ps}$, i.e. the effective delay $\delta$ due to the pulse front tilt is $4.4 \mathrm{ps}$. The latter permits obtaining the back-tilt angle $\varepsilon$, by simple trigonometry as follows:

$\varepsilon=\operatorname{atan}\left[\frac{\delta}{\Delta t \sin \theta}\right]$

which gives $\varepsilon=8.9^{\circ}$.

\section{DISCUSSION}

The characteristics of the compressed pulse were computed as a function of compressor easing (i.e. grating G2 angle). It was critical to quantify benefits of misalignment, i.e. the pulse front back-tilt, versus the pulse incremental broadening across its aperture. Pulse broadening causes loss of peak irradiance on the target.

Variable irradiance as a function of spot radius leads to a transversely inhomogeneous plasma-column in temperature and density. Inhomogeneities cause refraction and self-absorption, thus hindering the ASE process. Therefore, the current method proves incapable of addressing the full spatial and temporal complexity of pumping a plasma gain medium. This is subject of ongoing research, where higher-order effects are addressed. These are briefly introduced in the next paragraph.

\subsection{Higher-Order Effects}

The spectral phase of the propagating pulse, with respect to the relative frequency $\Omega=\omega$ - $\omega_{\mathrm{c}}$ (in $\mathrm{rad} / \mathrm{ps}$, or $\mathrm{rad} \mathrm{THz}$ ), can be expressed as a Taylor series (using $\Omega$ one has a Maclaurin series), as follows:

$\phi(\Omega)=\Phi_{c}+\Phi^{\prime} \Omega+\Phi^{\prime \prime} \frac{\Omega^{2}}{2}+\Phi^{\prime \prime \prime} \frac{\Omega^{3}}{6}+\cdots=P_{o}+G D D+T O D+\cdots$

which was decomposed as follows:

$P_{o}=\Phi_{c}+\Phi^{\prime} \Omega$

is the baseline pulse shape, i.e. with the zero-order term as the phase of the central frequency, which adds a constant, and the first-order term that adds a delay, but none of these affect the shape. The second-order group delay dispersion (GDD) is obtained from macroscopic parameters such as the wavelength $(\lambda$ in $\mathrm{mm}$ ), the grating constant $(d$ in $m m$ ), the diffraction angle $\left(\beta_{\lambda}\right)$, and the orthogonal grating separation $(x$ in $\mathrm{mm})$ as follows:

$G D D=x \cdot G V D=x \cdot \frac{\lambda^{3}}{2 \pi c^{2}}\left(\frac{\Omega^{2}}{d^{2} \cos ^{3} \beta_{\lambda}}\right)$

where GVD is the well-known group velocity dispersion, which in the present case was determined as $-31.1 \cdot 10^{-6} \mathrm{rad}$ $\mathrm{ps}^{2} / \mathrm{mm}$. The GDD introduces a frequency-dependent delay of the spectral components in the pulse. Differently from applications in the ultrafast regime, e.g. Ti:sapphire lasers, the generation of ps pulses, suitable for plasma lasing, relaxes the values, since the bandwidths involved are significantly smaller. The third-order dispersion (TOD) applies a quadratic phase across the pulse, but was neglected in the previous analysis because found less relevant. The ratio of third-order contribution over the second-order term, i.e. $\Phi^{3 / 2}$, was computed in the previous analysis, see Fig. 3. The $\Phi^{3 / 2}$ truncation ratio is as follows:

$\phi^{3 / 2}=\frac{\Delta \omega}{\omega_{c}}\left[1+\frac{\frac{\lambda}{d}\left(\frac{\lambda}{d}+\sin \alpha\right)}{1-\left(\frac{\lambda}{d}-\sin \alpha\right)^{2}}\right]$

The analysis has shown that the error done by truncating the series to second-order (linear chirp GDD) is as low as $0.12 \%$. Of course, this value is a bulk average over the entire pulse, and the regions at the periphery do experience the strongest effects. Thus local effects may get important, i.e. to better control the plasma pumping.

\section{CONCLUSIONS}

Pulse-front tilt to compensate TWE velocity at large target-irradiation angle, needed for short-wavelength lasing, was shown to be effective only if coupled with a strong front-end imaging/focusing component. The study shows that with 
approx. 10-fold front-end demagnification it is possible to obtain TWE speed-matching, for less than half degree compressor-grating tilt. The alternative technique of using large compressor tilting and modest imaging magnification is negatively affecting the performance. Indeed, concomitant loss of pulse peak power at the beam boundary can be very large. Variable irradiance as a function of beam radius, consequence of transverse pulse broadening, leads to a transversely inhomogeneous plasma-column in temperature and density. Inhomogeneities cause refraction and self-absorption, thus hindering the amplification process across the plasma length. It is noted that short wavelength and small bandwidth mitigate the broadening effect, and in the present case the best compromise is provided by the $\mathrm{Nd}$ :glass laser.

\section{REFERENCES.}

1. Bleiner, D. Saturation gain-length product during short-wavelength plasma lasing. Appl. Phys. Lett. 101, 0-3 (2012).

2. Masoudnia, L. \& Bleiner, D. Optimum electron temperature and density for short-wavelength plasma-lasing from nickel-like ions. Nucl. Inst. Methods Phys. Res. B 323, 59-70 (2014).

3. Nickles, P. V et al. Short Pulse X-Ray Laser at $32.6 \mathrm{~nm}$ Based on Transient Gain in Ne-like Titanium. Phys. Rev. Lett. 78, 2748 (1997).

4. Bleiner, D. \& Feurer, T. Pulse-front tilt for short-wavelength lasing by means of traveling-wave plasmaexcitation. Appl. Opt. 51, 8848-8853 (2012).

5. $\quad$ Tommasini, R. \& Fill, E. E. Generalized Linford formula. J. Opt. Soc. Am. B 17, 1665 (2000).

6. $\quad$ Pretzler, G., Kasper, A. \& Witte, K. J. Angular chirp and tilted light pulses in CPA lasers. Appl. Phys. B Lasers Opt. 70, 1-9 (2000).

7. Lin, J. Y. et al. Travelling wave chirped pulse amplified transient pumping for collisional excitation lasers. Opt. Commun. 166, 211-218 (1999).

8. Bowlan, P., Gabolde, P., Coughlan, M. A., Trebino, R. \& Levis, R. J. Measuring the spatiotemporal electric field of ultrashort pulses with high spatial and spectral resolution RETRIEVAL. 25, 81-92 (2008).

9. Pariente, G., Gallet, V., Borot, A., Gobert, O. \& Quéré, F. Space-time characterization of ultra-intense femtosecond laser beams. Nat. Photonics 10, 547-553 (2016).

10. Sacks, Z. \& Mourou, G. Adjusting pulse-front tilt and pulse duration by use of a single-shot autocorrelator. 26, 462-464 (2001).

11. Figueira, G., Cardoso, L., Lopes, N. \& Wemans, J. Mirrorless single-shot tilted pulse front autocorrelator. J. Opt. Soc. Am. B 22, 2709-2714 (2005).

12. Figueira, G. et al. Simultaneous measurement of pulse front tilt and pulse duration with a double trace autocorrelator. J. Opt. Soc. Am. B 36, 366 (2019).

13. Bleiner, D. \& Feurer, T. Fourier optics study of traveling-wave excitation at short-wavelength plasma-lasing. Springer Proc. Phys. 147, 35-38 (2014).

14. Staub, F., Bleiner, D., Jia, F. \& Balmer, J. E. Line-Focus Generation for X-ray Laser Pumping. 2-5

15. Bleiner, D., Balmer, J. E. \& Staub, F. Line focusing for soft x-ray laser-plasma lasing. Appl. Opt. 50, 6689-96 (2011). 